Imaging of High-Energy X-Ray Emission from Cryogenic Thermonuclear Fuel Implosions on the NIF

T. Ma

May 4, 2012

High-Temperature Plasma Diagnostics Conference Monterey, CA, United States

May 6, 2012 through May 10, 2012 
This document was prepared as an account of work sponsored by an agency of the United States government. Neither the United States government nor Lawrence Livermore National Security, LLC, nor any of their employees makes any warranty, expressed or implied, or assumes any legal liability or responsibility for the accuracy, completeness, or usefulness of any information, apparatus, product, or process disclosed, or represents that its use would not infringe privately owned rights. Reference herein to any specific commercial product, process, or service by trade name, trademark, manufacturer, or otherwise does not necessarily constitute or imply its endorsement, recommendation, or favoring by the United States government or Lawrence Livermore National Security, LLC. The views and opinions of authors expressed herein do not necessarily state or reflect those of the United States government or Lawrence Livermore National Security, LLC, and shall not be used for advertising or product endorsement purposes. 


\title{
Imaging of High-Energy X-Ray Emission from Cryogenic Thermonuclear Fuel Implosions on the NIF ${ }^{\text {a) }}$
}

\author{
T. Ma, ${ }^{1, b)}$ N. Izumi, ${ }^{1}$ R. Tommasini, ${ }^{1}$ D. K. Bradley, ${ }^{1}$ P. Bell, ${ }^{1}$ C. J. Cerjan, ${ }^{1}$ S. Dixit, ${ }^{1}$ \\ O. Jones, ${ }^{1}$ J. L. Kline, ${ }^{2}$ G. Kyrala, ${ }^{2}$ O. L. Landen, ${ }^{1}$ S. LePape, ${ }^{1}$ A. J. Mackinnon, ${ }^{1}$ H- \\ S. Park, ${ }^{1}$ R. R. Prasad, ${ }^{1}$ S. P. Regan, ${ }^{3}$ P. T. Springer, ${ }^{1}$ L. Suter, ${ }^{1}$ R. P. J. Town, ${ }^{1}$ S. \\ V. Weber, ${ }^{1}$ and S. H. Glenzer ${ }^{1}$
}

${ }^{1}$ Lawrence Livermore National Laboratory, Livermore, California 94550, USA
${ }^{2}$ Los Alamos National Laboratory, Los Alamos, New Mexico 87545, USA
${ }^{3}$ Laboratory for Laser Energetics, University of Rochester, New York 14623, USA

(Presented XXXXX; received XXXXX; accepted XXXXX; published online XXXXX)

Accurately assessing and optimizing the implosion performance of inertial confinement fusion capsules is a crucial step to achieving ignition on the NIF. We have applied differential filtering (matched Ross filter pairs) to provide spectrally resolved time-integrated absolute x-ray self-emission images of the imploded core of cryogenic layered targets. Using bremsstrahlung assumptions, the measured absolute x-ray brightness allows for the inference of electron temperature, electron density, hot spot mass, mix mass, and pressure.

\section{INTRODUCTION}

Current inertial confinement fusion (ICF) experiments ${ }^{1,2}$ conducted on the National Ignition Facility (NIF) ${ }^{3}$ seek to indirectly drive a spherical implosion, compressing and igniting a deuterium-tritium fuel. This DT fuel capsule is cryogenically prepared as a solid ice layer surrounded by a low-Z ablator material. Ignition will occur when the hot spot approaches sufficient temperature $(\sim 3-4 \mathrm{keV})$ and $\rho \mathrm{R}\left(\sim 0.3 \mathrm{~g} / \mathrm{cm}^{2}\right)$ such that alpha deposition can further heat the hot spot and generate a selfsustaining burn wave.,

During the implosion, the fuel mass becomes hot enough to emit large amounts of x-ray radiation, the spectra and spatial variation of which contains key information that can be used to evaluate the implosion performance. The Ross filter diagnostic employs differential filtering ${ }^{6,7}$ to provide spectrally resolved, time-integrated, absolute x-ray self-emission images of the imploded core of cryogenic layered targets.

\section{THE ROSS FILTER DIAGNOSTIC}

\section{A. Diagnostic setup}

The Ross filter diagnostic is a set of filters matched in $\mathrm{Z}$ and thickness to provide time-integrated $\mathrm{x}$-ray emission within narrow energy bands. The design currently being run on NIF shots uses five filter materials arranged in a repeating pattern mounted on an aluminum frame, coupled to a pinhole array, recording onto imaging plate (see Fig. 1(a)). Those five filters are listed in Fig. 1(b). The filter materials and thicknesses have been carefully chosen such that the transmitted x-ray spectra through the individual filters (Fig. 1(c)) are equivalent, except in the spectral region between their respective K-edges.

The five filter materials provide four energy bands when the images through the individual filters are subtracted from each other: $17.5 \mu \mathrm{m} \mathrm{Cu}+1525 \mu \mathrm{m}$ Kapton paired with the $50 \mu \mathrm{m} \mathrm{V}+$ $1525 \mu \mathrm{m}$ Kapton gives a 5.5 - $9 \mathrm{keV}$ channel; $24 \mu \mathrm{m}$ Ge coated on $200 \mu \mathrm{m}$ graphite $+1525 \mu \mathrm{m}$ Kapton paired with the $17.5 \mu \mathrm{m}$ $\mathrm{Cu}+1525 \mu \mathrm{m}$ Kapton gives a $9-11 \mathrm{keV}$ channel; $7 \mu \mathrm{m} \mathrm{Mo}+$ $1525 \mu \mathrm{m}$ Kapton paired with the $24 \mu \mathrm{m}$ Ge coated on $200 \mu \mathrm{m}$ graphite $+1525 \mu \mathrm{m}$ Kapton gives a $11-20 \mathrm{keV}$ channel; and $2525 \mu \mathrm{m}$ Kapton paired with the $7 \mu \mathrm{m}$ Mo $+1525 \mu \mathrm{m}$ Kapton gives a $>20 \mathrm{keV}$ channel. The width of the energy bands is chosen as a trade off between spectral resolution and capturing enough photons, particularly at the higher photon energies, where the emission from the implosion follows the bremsstrahlung relation and falls exponentially with energy.

The recording medium used is the Fuji SR-type image plate detector. Imaging plates are sensitive over a wide x-ray energy range, provide reasonable spatial resolution, are robust against EMP and high neutron yield (up to $\sim$ mid $10^{15}$ ), and are much simpler to process than traditional emulsion films.

The Ross filter diagnostic sits in a region surrounding the micro-channel plate of the NIF GXD (Gated X-Ray Detector) ${ }^{8}$ or hGXI (Hardened Gated X-Ray Imager), ${ }^{9}$ and shares the same pinhole array. In most cases, the distance from aimpoint to pinhole is $80 \mathrm{~mm}$ (on the equatorial 90-78 DIM) or $100 \mathrm{~mm}$ (on the polar 00-00 DIM), and pinhole plane to the image plate detector is $1118 \mathrm{~mm}$, resulting in a magnification of $14 \mathrm{x}$ and $11 \mathrm{x}$, respectively. The pinholes are of $10 \mu \mathrm{m}$ diameter, in a $75 \mu \mathrm{m}$ thick Ta substrate. Two sets of $500 \mu \mathrm{m}$ thick Ta collimators of $150 \mu \mathrm{m}$ diameter, further reduce the neutron and hard x-ray background (from the hohlraum emission) incident on the image plate detector.

\section{B. Absolute calibration}

The spectral response sensitivity curve of the SR image plate is taken from Maddox et $a l^{7}$, with a calibration factor of $7.82 \mathrm{MeV} / \mathrm{PSL}$, as determined by a recent calibration with a ${ }^{109} \mathrm{Cd}$ source. Image fade rates are also taken from Maddox et al.

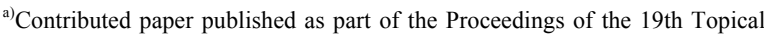
Conference on High-Temperature Plasma Diagnostics, Monterey, California, May, 2012.

b) Author to whom correspondence should be addressed: ma8@1lnl.gov 
(a)

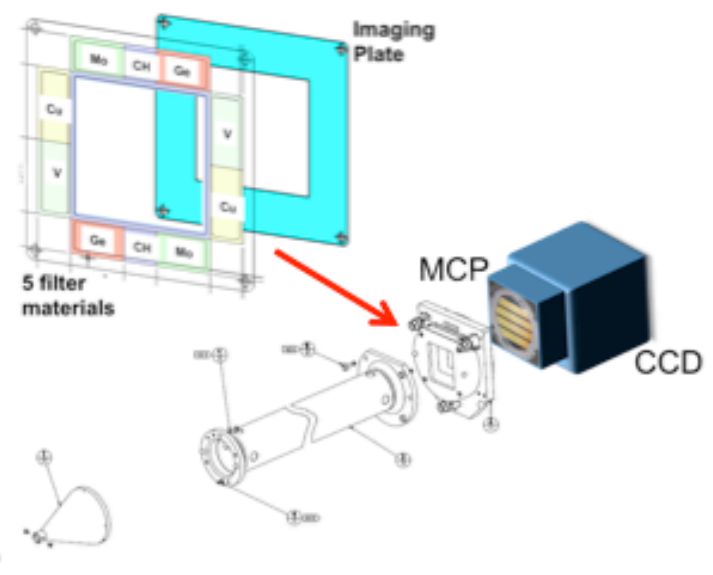

(b)

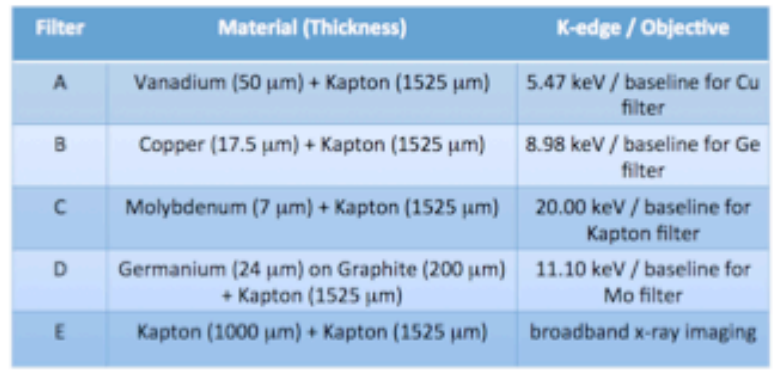

(c)

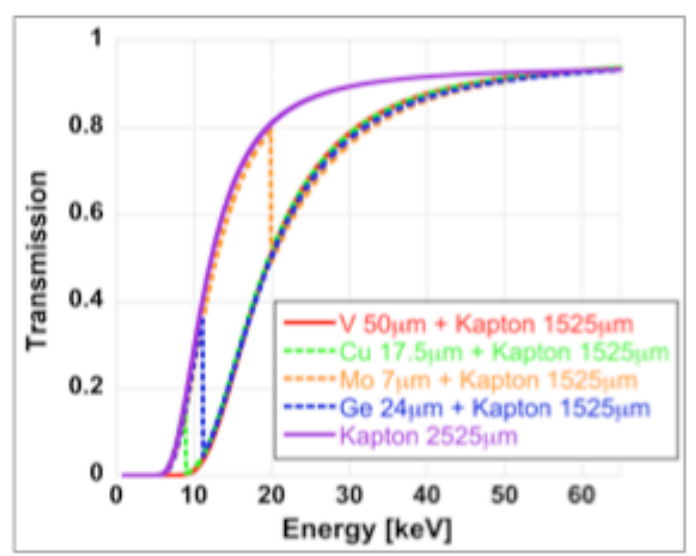

FIG. 1. (Color online). (a) Schematic of the Ross filter diagnostic, consisting of five filter materials arranged in an array. An imaging plate sits directly behind the filter assembly. (b) Each of the filter materials and their thickness in the Ross build, with their accompanying k-edge and objectives. (c) The transmission curves of each of the filters used in the assembly.

The transmission and uniformity of the filters in the assembled Ross array were calibrated by measuring the signal level transmitted through the filters at two energies (Ag Ka at 22 $\mathrm{keV}$ and $\mathrm{Zr} \mathrm{Ka}$ at $15 \mathrm{keV}$ ) using an $\mathrm{x}$-ray source. An image plate was directly exposed to the $\mathrm{x}$-ray beam at the nominal settings and duration as a filter measurement to serve as a flat field. The same image plates were then used to measure the x-ray signal through a Ross filter array that was placed directly in front of the IP. The intensity measured through the filter was divided by the intensity measured without the filter to provide a transmission measurement. The uniformity of all the filters was found to be

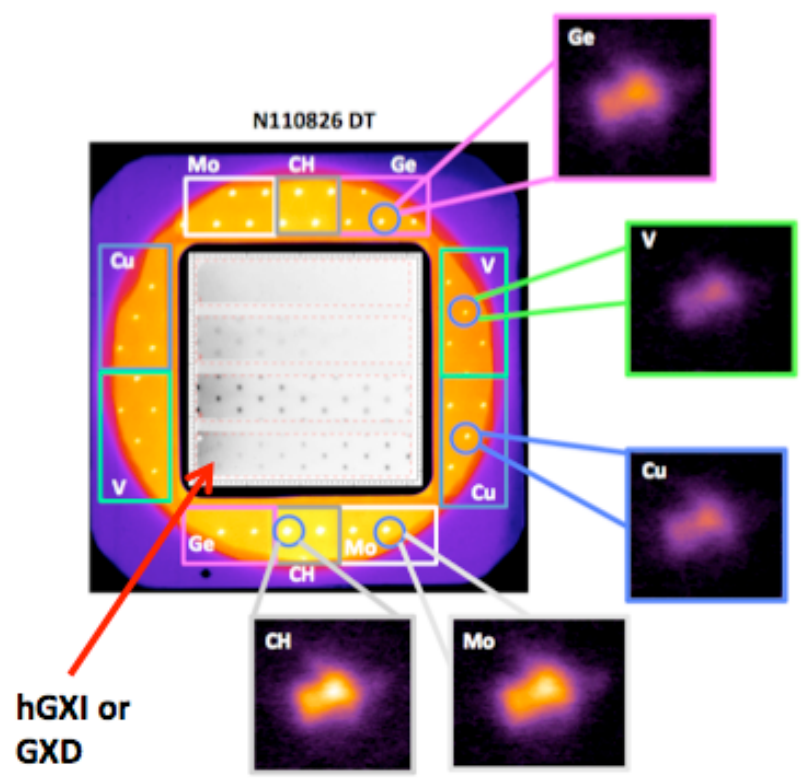

FIG. 2. (Color online). Raw differentially filtered images from DT shot N110826.

better than $2 \%$ averaged across the area of the filter. A second method was also used to verify filter thicknesses. Raw filter material (before cutting and assembly onto a filter frame) was weighed with a high accuracy microscale. Using the tabulated density for the material (and assuming no contaminants), the $\rho \mathrm{R}$ and thickness of the material could be calculated. Further, the raw materials were measured using a Heidenhain thickness gauge. A given sample was measured in its four corners and at various locations across its surface to provide an average thickness and estimated error bars. The filter thicknesses measured from the Heidenhain and microscale methods are in very good agreement. Filter thicknesses were found to be within $2 \%$ of the specified thicknesses.

\section{RESULTS AND DISCUSSION}

An example of the raw images obtained using the Ross Pair Filter diagnostic for a cryogenic DT shot is shown in Fig. 2. The $\mathrm{x}$-ray spectrum can then be derived from the data by subtracting the images associated with a Ross pair filter set, and converting that signal to absolute x-ray energy in the given channel. Figure 3 shows the predicted post-shot spectrum for a DT implosion, with the energy bins captured by the Ross filters shaded. The bulk of the x-ray emission emitted by the hot spot will be bremsstrahlung continuum ${ }^{5}$, for which emissivity scales as:

$$
P_{\text {brem }} \propto \frac{n^{2} Z^{2}}{T^{1 / 2}} \exp \left(-\frac{h v}{k T}\right)
$$

As can be seen in Fig. 3, the X-ray spectral emission peaks at $\sim 10$ $\mathrm{keV}$. The cut off on the low energy side is due to attenuation in the dense ablator shell, while fall off on the high energy side follows the bremsstrahlung relation. Energy below $6 \mathrm{keV}$ in the simulation is due to hohlraum emission. The K-edge due to a $\mathrm{Ge}$ dopant in the ablator can be seen at $11 \mathrm{keV} .{ }^{10}$ Then, equating the energies in the individual Ross spectral bins to radiated bremsstrahlung, we can reconstruct the x-ray spectrum.

In practice, this step is complicated by the fact that the filter pairs are typically not a perfect match, i.e., there remains a 


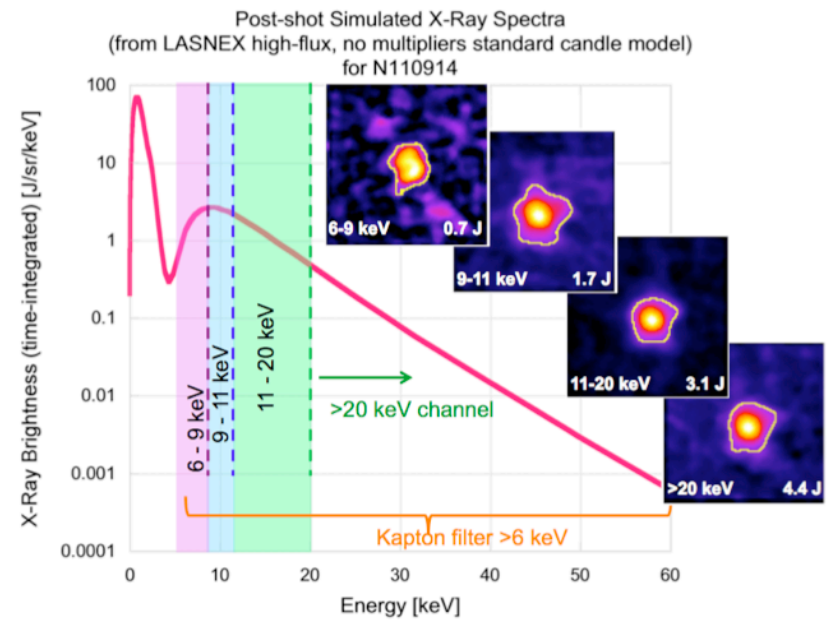

FIG. 3. (Color online). The simulated integrated x-ray spectrum for the DT implosion N110914. The shaded regions show the energy bins covered by the Ross filter pairs, while the corresponding images give spatial and absolute $\mathrm{x}$ ray energy information for each spectral band.

residual sensitivity outside the Ross pair energy band. Thus, there is a contribution to the measured signal intensity from either the lower energy or higher energy part of the x-ray spectrum that must be corrected for.

Bremsstrahlung falls exponentially as a function of photon energy, with the slope of the fall-off dependent on plasma temperature. The ratio of $\mathrm{x}$-ray emission through various channels of the Ross filters gives us the bremsstrahlung slope, and hence the hot spot burn-weighted electron temperature (because this is a time-integrated diagnostic, the peak emission will be emphasized). Further, because the Ross diagnostic is absolutely calibrated, the measured x-ray brightness can be equated to an absolute bremsstrahlung emission level, which in turn reveals information about the plasma density. The electron temperature can typically be determined to $\pm 300 \mathrm{eV}$, while electron densities are $\pm 30 \%$. The hot spot pressure can then be

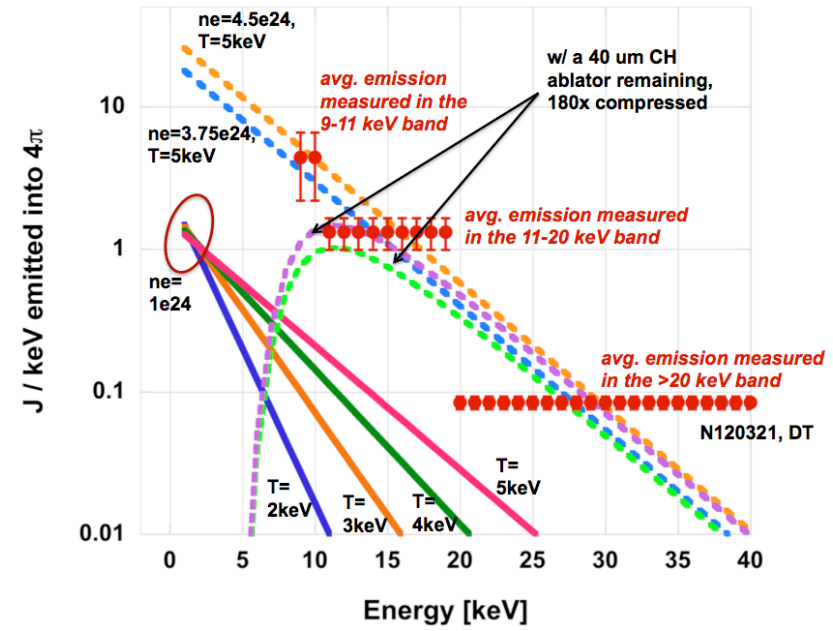

FIG. 4. (Color online). The absolutely calibrated ratio of $x-$ ray continuum emission provides a temperature and density measurement. Solid lines show the trend the bremsstrahlung continuum follows as a function of temperature, at a given electron density. Dashed lines show the range of ne and $\mathrm{T}$ that fit a set of average x-ray emission measured using the Ross filters for DT implosion N120321. inferred using the simple relation of $\mathrm{P}=2 \mathrm{nkT}$. The Ross diagnostic shows that present implosions achieve pressures of $>150$ Gbar. Pressures inferred in this manner have been found to be within $30 \%$ of a $3 \mathrm{D}$ model that attempts to fit the full set of diagnostic measurements. ${ }^{11}$

A concern at the lower photon energies is the compressed ablator shell, which absorbs some of the radiation before it can escape the hot spot. Therefore, assumptions must be made about how much mass is remaining, and how strongly compressed it is in order to reconstruct the original bremsstrahlung spectrum emitted by the hot spot. Also, small amounts of ablator that mix into the hot spot will actually increase the x-ray bremsstrahlung intensity due to the $Z^{2}$ dependence of the bremsstrahlung emission (the ablator is typically $\mathrm{CH}$ with some higher $\mathrm{Z}$ dopant). This can actually be used as a mix mass diagnostic. In this case, the "clean" DT electron density must be approximated from the measured values by the neutron diagnostics, and the x-ray continuum due to this hot mass of DT can be calculated. Any excess measured $\mathrm{x}$-ray brightness can then be attributed to $\mathrm{CH}(+$ additional high- $Z$ ablator) bremsstrahlung.

The differential filtering provided by the Ross filter diagnostic limits the spectral range of imaging. The absolutely measured brightness of bremsstrahlung within these limited energy bands allows for the inference of burn-weighted electron temperature, density, pressure, and mix mass.

\section{ACKNOWLEDGMENTS}

This work performed under the auspices of the U.S. Department of Energy by Lawrence Livermore National Laboratory under Contract DE-AC52-07NA27344.

${ }^{1}$ S. H. Glenzer et al., Plasma Phys. Control. Fusion 54, 045013 (2012).

${ }^{2}$ M. J. Edwards et al., Phys. Plasmas 18, 051003 (2011).

${ }^{3}$ G. H. Miller, E. I. Moses, and C. R. Wuest, Nucl. Fusion 44, 228 (2004).

${ }^{4}$ J. Nuckolls, L. Wood, A. Thiessen, and G. Zimmerman, Nature 239, 139 (1972).

${ }^{5}$ S. Atzeni and J. Meyer-Ter-Vehn, The Physics of Inertial Fusion Oxford Science Publications, Clarendon, Oxford, 2004.

${ }^{6}$ P. A. Ross, Phys. Rev. 28, 425 (1926).

${ }^{7}$ B. R. Maddox, H. S. Park, B. A. Remington, N. Izumi, S. Chen, C. Chen, G. Kimminau, Z. Ali, M. J. Haugh, and Q. Ma, Rev. Sci. Instrum. 82, 023111 (2011).

${ }^{8}$ John A. Oertel et al., Rev. Sci. Instrum. 77, 10E308 (2006).

${ }^{9}$ S. Glenn et al., Rev. Sci. Instrum. 81, 10E539 (2010).

${ }^{10}$ B. A. Hammel, M. J. Edwards, S. W. Haan, M. M. Marinak, M. Patel, H. Robey, and J Salmonson, J. Phys.: Conf. Ser. 112, 022007 (2008).

${ }^{11} \mathrm{~T}$. Ma, presented at the $53^{\text {rd }}$ Annual Meeting of the APS Division of Plasma Physics, Salt Lake City, UT, 14-18 November 2011. 